\title{
Multi-platform profiling of over 2000 sarcomas: Identification of biomarkers and novel therapeutic targets
}

\author{
Sujana Movva ${ }^{1, *}$, Wenhsiang Wen ${ }^{2, *}$, Wangjuh Chen ${ }^{2}$, SherriZ. Millis ${ }^{2}$, Zoran Gatalica ${ }^{2}$, \\ Sandeep Reddy ${ }^{2}$, Margaret von Mehren ${ }^{1}$, Brian A. Van Tine ${ }^{3}$ \\ ${ }^{1}$ Fox Chase Cancer Center, Philadelphia, PA, USA \\ ${ }^{2}$ Caris Life Sciences, Phoenix, AZ, USA \\ ${ }^{3}$ Washington University in St. Louis, St. Louis, MO, USA \\ *These authors have contributed equally to this work \\ Correspondence to: \\ Sujana Movva, e-mail: sujana.movva@fccc.edu \\ Keywords: sarcoma, biomarkers, targeted therapies, DNA sequencing, protein expression \\ Received: February 03, $2015 \quad$ Accepted: March 07, $2015 \quad$ Published: March 26, 2015
}

\section{ABSTRACT}

Background: Drug development in sarcoma has been hampered by the rarity and heterogeneity of the disease and lack of predictive biomarkers to therapies. We assessed protein expression and gene alterations in a large number of bone and soft tissue sarcomas in order to categorize the molecular alterations, identify predictive biomarkers and discover new therapeutic targets. Methods: Data from sarcoma specimens profiled for protein expression, gene amplification/translocation and DNA sequencing was reviewed. Results: 2539 sarcoma specimens of 22 subtypes were included. TOPO2A was the most overexpressed protein at $52.8 \%$. There was overexpression or loss of other sarcoma relevant proteins such as SPARC, PTEN and MGMT. Approximately $50 \%$ of the sarcomas expressed PD-L1 by IHC and presented with PD-1+ TILs, notably the LMS, chondrosarcomas, liposarcomas and UPS. Gene amplification/rearrangement of ALK, CMYC, HER2, PIK3CA, TOPO2A and CMET was relatively uncommon. EGFR gene amplification occurred at a rate of $16.9 \%$. DNA sequencing of 47 genes identified mutations in $47 \%$ of the samples. The most commonly mutated genes were TP53 (26.3\%) and BRCA2 (17.6\%). Overexpression of TOPO2A was associated with TP53 mutation $(P=0.0001)$. Conclusion: This data provides the landscape of alterations in sarcoma. Future clinical trials are needed to validate these targets.

\section{INTRODUCTION}

Sarcomas are a rare, heterogeneous group of mesenchymal tumors. With over 100 subtypes, a single therapeutic strategy for this group of cancers is unfeasible. For patients with advanced disease, the selection of therapy is based on the specific sarcoma subtype as well as the patient's fitness to receive aggressive chemotherapy. With this approach, median overall survival for patients with advanced bone and soft tissue sarcomas (STS) remains under two years [1]. Sarcomas can be classified according to the genetic alterations involved in their development: those with oncogenic somatic mutations [e.g. gastrointestinal stromal tumors (GIST)], those with DNA copy number alterations (e.g. dedifferentiated liposarcomas), and those with recurrent chromosomal translocations resulting in abnormal fusion proteins (e.g. synovial sarcomas). More commonly, sarcomagenesis is a result of complex chromosomal abnormalities, as in the case of leiomyosarcomas and high grade undifferentiated pleomorphic sarcomas [2].

Analyses of genetic alterations in sarcoma have generally focused on particular subtypes $[3,4]$ and frequently employ limited methodologies. Data from large scale, systematic genomic profiling of sarcomas is limited. Barretina and colleagues characterized 207 sarcoma specimens, including seven different subtypes, by DNA sequence, copy number alterations and mRNA expression. They were able to identify potentially druggable PIK3CA mutations in $18 \%$ of myxoid liposarcomas [5]. Conversely, a separate series, by Cote 
and colleagues found that when solely using DNA hotspot analysis or whole exome sequencing, mutations were rare and there was no pattern of alterations noted within sarcoma subtypes [6].

Protein biomarkers have been studied for many decades, in an attempt to predict which tumors will respond to what therapies. This is best highlighted in the breast cancer field, where the proteins estrogen receptor (ER), progesterone receptor (PR), and human epidermal growth factor receptor (HER2) have been well established as prospective treatment response biomarkers $[7,8]$. To date, a large analysis for common chemotherapy associated biomarkers has not been performed across a large group of sarcomas. A detailed mapping of the therapy associated biomarkers will allow for identification of both targets and histologies that may respond to a particular drug, in order to design better clinical trials [9].

Using a registry of 2539 patients with bone and soft tissue sarcomas we have catalogued changes in protein expression, gene amplification/translocation, and somatic mutations. Herein we describe these findings with the goal of systematically characterizing the molecular alterations in a variety of sarcoma subtypes, identifying potential biomarkers of sensitivity to chemotherapy and targeted agents, and potentially discovering novel therapeutic targets.

\section{RESULTS}

\section{Population}

2539 sarcoma specimens encompassing 61 bone and STS subtypes were profiled. These included 22 standard histologies, and an "other" $(n=454)$ category, that included sarcoma NOS $(n=251)$ and spindle cell tumor $(n=103)$, as well as 37 sarcoma subtypes that had fewer than 10 cases. The most common histology was leiomyosarcoma (LMS) $(n=751)$ with 401 of these uterine, followed by liposarcoma $(n=220)$ and undifferentiated pleomorphic sarcoma [UPS (formally malignant fibrous histiocytoma) $(n=166)]$. Of the 77 angiosarcomas, 14 were of breast origin. 862 samples were known to be from a metastatic site. The median age of the population was 53 (range: $1-92$ ). $62 \%$ of the cases were from females. Up to 2434 samples were profiled by IHC, 1048 by FISH/CISH, 591 by NGS and 1250 by Sanger sequencing. 530 samples were profiled on all three platforms (IHC, FISH/CISH and either NGS or Sanger sequencing).

\section{Protein biomarkers}

TOPO2A overexpression, an anthracycline associated response biomarker, was noted in 52.8\% of the sarcomas and in greater than $60 \%$ of MPNST, angiosarcoma, LMS, rhabdomyosarcoma and UPS. High expression of TOPO2A has been previously reported at a rate of $50 \%$ in STS, when median percentage was chosen as the cutoff to discriminate between high and low expressing tumors, rather than criteria previously described in breast cancer [10,11]. Overexpression of serum protein acidic and rich in cysteine (SPARC), a biomarker for albumin bound paclitaxel, was seen in $35.9 \%$ of the cases, especially in over $60 \%$ of epithelioid hemangioendothelioma (EHE) and chondrosarcoma (notably conventional chondrosarcoma), as well as $48.7 \%$ of angiosarcomas. A previous study of SPARC expression by IHC, noted a rate of high SPARC staining in $56 \%$ of specimens, but given the small sample size $(n=27)$, specific histology correlations could not be made [12]. Low MGMT expression, a temozolomide associated biomarker, was noted in a variety of sarcomas including alveolar soft part sarcoma (21 ASPS), desmoid, EHE, perivascular epithelioid cell tumor (PEComa), endometrial stromal sarcoma (ESS), giant cell tumor, liposarcoma, LMS, malignant peripheral nerve sheath tumor (MPNST), osteosarcoma and UPS. There was low expression of MGMT in $65.3 \%$ of the sarcomas overall. Previous studies have only considered nuclear staining positive, and have reported a much lower rate of MGMT loss in LMS and other STS subtypes, therefore validation of a particular method is required to determine the predictive value in STS [13]. PTEN loss was seen in $38.6 \%$ of the sarcomas, most commonly in epithelioid sarcoma, chordoma, alveolar rhabdomyosarcoma and osteosarcoma. PTEN loss was only noted in $32.2 \%$ of non-uterine LMS and $37.6 \%$ of uterine LMS. Previous work on complex genomic sarcomas such as LMS, UPS and MPNST have reported rates of PTEN loss in $29 \%-44 \%$ of the sarcomas, $[14,15]$ but data on the expression of PTEN in rarer sarcomas is lacking. cKIT overexpression was noted in $28.5 \%$ of angiosarcoma, $19 \%$ of desmoplastic small round cell tumor (DSRCT) and $37.3 \%$ of Ewing's sarcoma. There were 2 cases of sclerosing rhabdomyosarcoma, both of which overexpressed cKIT. This is a rare sarcoma whose treatment paradigm is not yet clearly defined. PDGFRA was overexpressed in $22.1 \%$ of the sarcomas, including, $38.5 \%$ of angiosarcoma, $33.3 \%$ of liposarcoma, $33.3 \%$ of fibrosarcoma, $31.8 \%$ of Ewing's sarcoma, $30.8 \%$ of chondrosarcoma, $27.8 \%$ of osteosarcoma, $27.8 \%$ of UPS and $18.3 \%$ of non-uterine LMS. High PDGFRA expression has been described previously in many of these tumors [16-18]. In a series by Rodrigo and colleagues HER2 protein expression by IHC was negative in all sarcoma samples [10]. HER2 overexpression was noted in only one case in this series, an ESS, confirming that this is not an important pathway in sarcoma (Figure 1a and $1 \mathrm{~b}$ and Supplementary Table 1).

AR overexpression was seen in $18.3-54.2 \%$ of myxoid chondrosarcoma, DSRCT, pleomorphic and 
well-differentiated liposarcoma, ESS and LMS. ER overexpression was seen in $47.8 \%$ of ESS, $23.0 \%$ of non-uterine LMS, $60.3 \%$ of uterine LMS and $23.5 \%$ of PEComas (Figure 1c and Supplementary Table 1). The rates of ER overexpression in uterine LMS appear similar to previous studies [19, 20]. This data also confirms the rate of ER positivity in non-uterine LMS at approximately $20 \%$ with a much larger number of cases [21].

Overall, approximately $50 \%$ of the sarcomas expressed PD-L1 by IHC and presented with PD-1+ TILs, similar to other series [22]. Sarcomas with sufficient number of cases and overexpression of these proteins included LMS, chondrosarcoma, liposarcoma and UPS. Only 4 cases of synovial sarcoma were available for testing, 1 of which had simultaneous overexpression of PD-L1 and presence of PD-1+ TILs (Figure 1d and Supplementary Table 2). This rate is lower than published literature [22].

\section{FISH/CISH}

The most commonly amplified gene was EGFR at a rate of $16.9 \%$ overall. This was especially noted in histologies such as DSRCT, LMS, MPNST, osteosarcoma and UPS at a rate of $20 \%$ or higher. Smaller sarcoma series have suggested a lower rate of amplification overall $(3.5 \%)$, most commonly in UPS [23]. In series focusing on MPNST specifically, the rate of EGFR gene amplification by FISH was 28-37\% [24, 25]. None of the synovial sarcomas in our series demonstrated genomic amplification of $E G F R$, consistent with previous work [26]. Amplification of the ALK, $C M Y C, P I K 3 C A$ and TOPO2A genes were relatively uncommon events. $c M E T$ was amplified in 5-6\% of osteosarcoma and synovial sarcomas. HER2 was amplified in $5.6 \%$ of MPNST (Table 1).

\section{DNA sequencing}

591 samples were profiled by NGS and 1250 by Sanger sequencing. $47 \%$ of the samples had an identifiable mutation in 35 of the 47 genes analyzed. The most commonly mutated genes overall were TP53 (26.3\%) and BRCA2 (17.6\%). Histologies carrying mutations at a frequency of $\geq 5 \%$ included: angiosarcoma (APC, BRAF, GNA11, HRAS KDR, KRAS, $N R A S$ ), chondrosarcoma [IDH1 (conventional and unknown/other), PTEN (myxoid), cMET (conventional and mesenchymal)], desmoid (APC, CTNNB1, STK11), ESS (AKT1, cMET, FGFR2, GNAS, KRAS, RET, $S M O)$, Ewing's sarcoma (APC, ATM, HNF1A, PTEN), fibrosarcoma $(K R A S)$, giant cell tumor $(K R A S)$, myxoid liposarcoma (AKT1, ATM, cMET, JAK3, PIK3CA, $P T E N)$, dedifferentiated liposarcoma (HNF1), well- differentiated liposarcoma (cMET), LMS (BRCA2, RB1), MPNST (BRAF V600E), osteosarcoma (cKIT, FLT3), rhabdomyosarcoma [FLT3 (pleomorphic), PIK3CA (uknown/other), PTEN (unknown/other), PTPN11 (alveolar), SMARCB1 (unknown/other)], solitary fibrous tumor (cKIT, PDGFRA, PIK3CA, STK11), synovial sarcoma (ABL1, ATM, BRAF, $C K I T, K D R, M L H 1)$ and UPS (KDR, PIK3CA). BRCA2 mutations were seen in $17 \%$ of LMS, both uterine and non-uterine. PTEN and $R B 1$ mutations were noted exclusively in non-uterine LMS and not in those of uterine origin. NRAS mutations were detected in $20 \%$ of non-breast angiosarcomas, and were not found in those of breast origin. The PIK3CA mutations noted in liposarcoma (5 cases) were found in 4 myxoid liposarcomas and in 1 high grade pleomporphic liposarcoma. No EGFR mutations were detected in our series (Figure $2 \mathrm{a}-\mathrm{d}$ and Supplementary Table 3). One case of MPNST carried a G12V KRAS mutation. We detected BRAF, PTEN, p53 and NRAS mutations in angiosarcoma specimens, not previously described in the literature [4].

\section{Biomarker associations}

There was relatively low concordance across platforms for individual genes or proteins (Table 2). For example, cKIT overexpression by IHC was infrequently associated with $c K I T$ mutations. This is in contrast to the scenario in GIST, where more than $80 \%$ of cases carry an activating mutation in the KIT gene [27]. In our series, overexpression of TOPO2A by IHC was not associated with TOPO2A gene amplification, similar to some series in breast cancer and in contrast to others [28, 29]. We also examined the association of TP53 or PIK3CA mutations with other alterations (Table 3 and Table 4). We noted that $85.8 \%$ of samples demonstrated both TOPO2A expression by IHC and TP53 mutation ( $P$ value $=$ $0.0001)$. Three patient tumor samples had both a PIK3CA mutation and PTEN loss by IHC (myxoid liposarcoma, rhabdomyosarcoma and sarcoma, NOS). Two patient samples had EGFR amplification and $K R A S$ mutation (1 MFH and 1 ESS).

\section{DISCUSSION}

This data set represents the largest number of sarcoma cases molecularly profiled to date. We observed that TOPO2A was overexpressed in approximately $50 \%$ of sarcomas, without associated gene amplification, most commonly in the angiosarcomas, LMS, MPNST, rhabdomyosarcoma and UPS. Indeed, amplification of the TOPO2A gene has not reliably predicted increased protein levels of TOPO2A in other cancers $[11,29,30]$. 


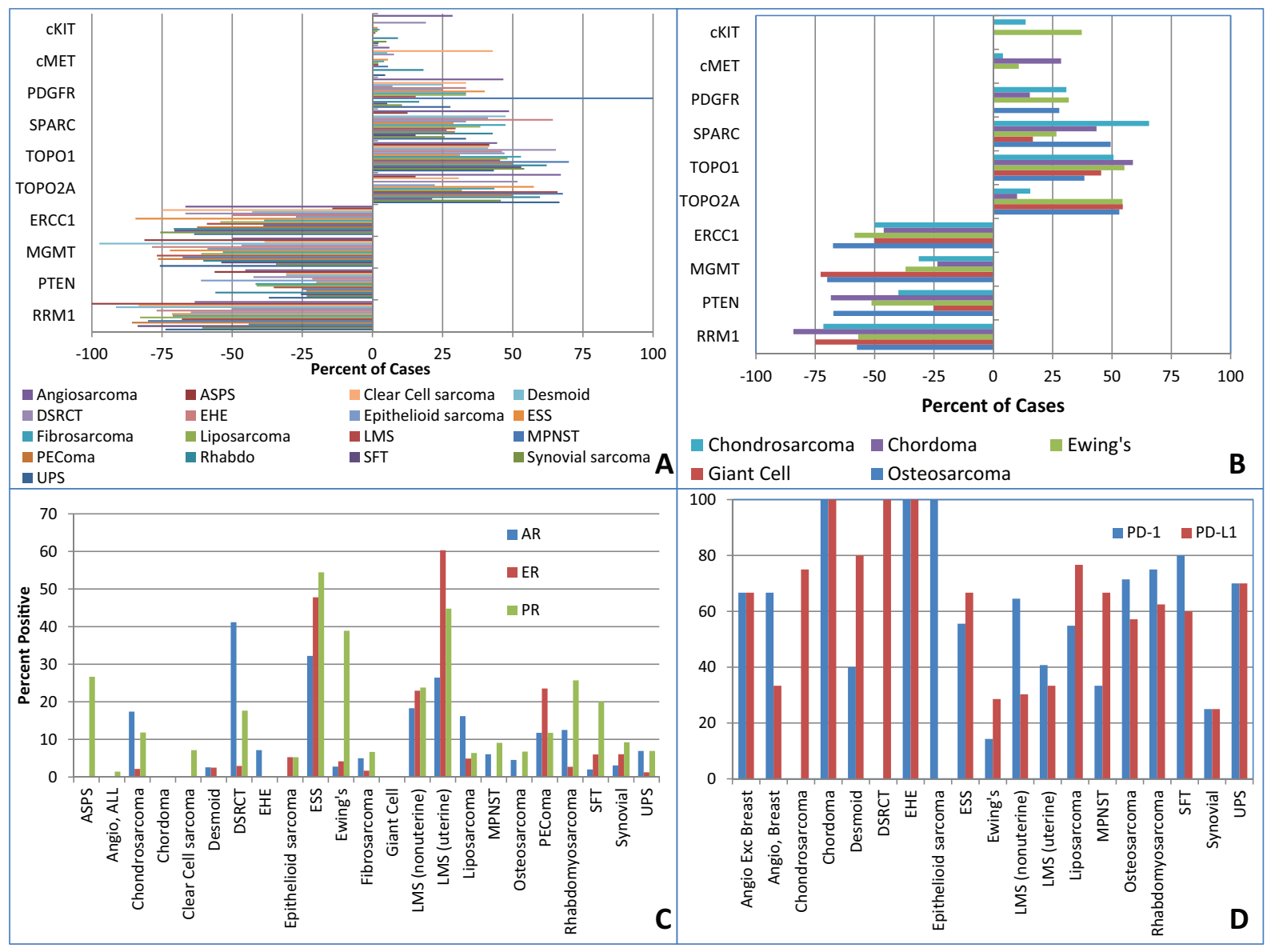

Figure 1: Protein biomarker expression by IHC. A. Soft tissue sarcomas B. Bone sarcomas C. Hormone receptors D. PD-1, PD-L1. ASPS = alveolar soft part sarcoma, DSRCT $=$ desmoplastic small round cell tumor, EHE = epithelioid hemangioendothelioma $\mathrm{ESS}=$ endometrial stromal sarcoma, SFT = solitary fibrous tumor, LMS = leiomyosarcoma, UPS = undifferentiated pleomorphic sarcoma, MPNST = malignant peripheral nerve sheath tumor, PEComa = perivascular epithelioid cell tumor.

Table 1: FISH/CISH by histology

\begin{tabular}{|c|c|c|c|c|}
\hline \multirow[b]{2}{*}{ Histology } & & \multicolumn{3}{|c|}{ Gene $^{* *}$} \\
\hline & & ISH cMET & ISH EGFR & ISH HER2 \\
\hline \multirow[t]{3}{*}{ All $(n=2539)$} & Total Positive & 20 & 181 & 9 \\
\hline & Total Cases & 761 & 1072 & 925 \\
\hline & $\%$ Positive & 2.6 & 16.9 & 1.0 \\
\hline \multirow[t]{3}{*}{ Angiosarcoma $(n=77)$} & Total Positive & 0 & 1 & 0 \\
\hline & Total Cases & 26 & 34 & 33 \\
\hline & $\%$ Positive & 0.0 & 2.9 & 0.0 \\
\hline \multirow[t]{3}{*}{ Chondrosarcoma $(n=97)$} & Total Positive & 1 & 2 & 1 \\
\hline & Total Cases & 32 & 24 & 42 \\
\hline & $\%$ Positive & 3.1 & 8.3 & 2.4 \\
\hline
\end{tabular}

(Continued) 


\begin{tabular}{|c|c|c|c|c|}
\hline \multirow[b]{2}{*}{ Histology" } & & \multicolumn{3}{|c|}{ Gene $^{* *}$} \\
\hline & & \multirow{2}{*}{$\begin{array}{l}\text { ISH cMET } \\
0\end{array}$} & \multirow{2}{*}{$\begin{array}{l}\text { ISH EGFR } \\
1 \\
\end{array}$} & \multirow{2}{*}{$\begin{array}{l}\text { ISH HER2 } \\
0 \\
\end{array}$} \\
\hline Clear cell sarcoma $(n=16)$ & Total Positive & & & \\
\hline & Total Cases & 4 & 8 & 4 \\
\hline & $\%$ Positive & 0.0 & 12.5 & 0.0 \\
\hline \multirow[t]{3}{*}{$\operatorname{DSRCT}(n=8)$} & Total Positive & 0 & 2 & 0 \\
\hline & Total Cases & 11 & 10 & 15 \\
\hline & $\%$ Positive & 0.0 & 20.0 & 0.0 \\
\hline \multirow[t]{3}{*}{$\operatorname{ESS}(n=91)$} & Total Positives & 0 & 4 & 1 \\
\hline & Total Cases & 33 & 50 & 31 \\
\hline & $\%$ Positive & 0.0 & 8.0 & 3.2 \\
\hline \multirow[t]{3}{*}{ Ewing's sarcoma $(n=83)$} & Total Positive & 0 & 2 & 0 \\
\hline & Total Cases & 23 & 30 & 27 \\
\hline & $\%$ Positive & 0.0 & 6.7 & 0.0 \\
\hline \multirow[t]{3}{*}{ Fibrosarcoma $(n=63)$} & Total Positive & 0 & 5 & 0 \\
\hline & Total Cases & 21 & 33 & 21 \\
\hline & $\%$ Positive & 0.0 & 15.2 & 0.0 \\
\hline \multirow[t]{3}{*}{ Giant cell tumor $(n=13)$} & Total Positive & 0 & 2 & 0 \\
\hline & Total Cases & 3 & 7 & 4 \\
\hline & $\%$ Positive & 0.0 & 28.6 & 0.0 \\
\hline \multirow[t]{3}{*}{ Liposarcoma $(n=220)$} & Total Positive & 3 & 14 & 0 \\
\hline & Total Cases & 62 & 80 & 93 \\
\hline & $\%$ Positive & 4.8 & 17.5 & 0.0 \\
\hline \multirow[t]{3}{*}{ Myxoid $(n=46)$} & Total Positive & 1 & 0 & 0 \\
\hline & Total Cases & 14 & 15 & 18 \\
\hline & $\%$ Positive & 7.1 & 0.0 & 0.0 \\
\hline \multirow[t]{3}{*}{ Dedifferentiated $(n=77)$} & Total Positive & 0 & 1 & 0 \\
\hline & Total Cases & 24 & 28 & 41 \\
\hline & $\%$ Positive & 0.0 & 3.6 & 0.0 \\
\hline \multirow[t]{3}{*}{ Well-differentiated $(n=31)$} & Total Positive & 0 & 5 & 0 \\
\hline & Total Cases & 11 & 12 & 13 \\
\hline & $\%$ Positive & 0.0 & 41.7 & 0.0 \\
\hline
\end{tabular}

(Continued) 


\begin{tabular}{|c|c|c|c|c|}
\hline \multirow[b]{2}{*}{ Histology* } & & \multicolumn{3}{|c|}{ Gene $^{* *}$} \\
\hline & & \multirow{2}{*}{$\begin{array}{l}\text { ISH cMET } \\
2 \\
\end{array}$} & \multirow{2}{*}{$\begin{array}{l}\text { ISH EGFR } \\
5 \\
\end{array}$} & \multirow{2}{*}{$\begin{array}{l}\text { ISH HER2 } \\
0 \\
\end{array}$} \\
\hline Pleomorphic $(n=30)$ & Total Positive & & & \\
\hline & Total Cases & 7 & 8 & 10 \\
\hline & $\%$ Positive & 28.6 & 62.5 & 0.0 \\
\hline \multirow[t]{3}{*}{ Other/Unknown $(n=36)$} & Total Positive & 0 & 3 & 0 \\
\hline & Total Cases & 6 & 17 & 11 \\
\hline & $\%$ Positive & 0.0 & 17.6 & 0.0 \\
\hline \multirow[t]{3}{*}{ LMS (nonuterine) $(n=350)$} & Total Positive & 4 & 27 & 3 \\
\hline & Total Cases & 112 & 128 & 137 \\
\hline & $\%$ Positive & 3.6 & 21.1 & 2.2 \\
\hline \multirow[t]{3}{*}{ LMS (uterine) $(n=401)$} & Total Positive & 4 & 43 & 1 \\
\hline & Total Cases & 96 & 220 & 106 \\
\hline & $\%$ Positive & 4.2 & 19.5 & 0.9 \\
\hline \multirow[t]{3}{*}{ MPNST $(n=36)$} & Total Positive & 0 & 4 & 1 \\
\hline & Total Cases & 13 & 14 & 18 \\
\hline & $\%$ Positive & 0.0 & 28.6 & 5.6 \\
\hline \multirow[t]{3}{*}{ Osteosarcoma $(n=95)$} & Total Positive & 1 & 9 & 0 \\
\hline & Total Cases & 18 & 46 & 24 \\
\hline & $\%$ Positive & 5.6 & 19.6 & 0.0 \\
\hline \multirow[t]{3}{*}{ PEComa $(n=17)$} & Total Positive & 0 & 2 & 0 \\
\hline & Total Cases & 9 & 7 & 9 \\
\hline & $\%$ Positive & 0.0 & 28.6 & 0.0 \\
\hline \multirow[t]{3}{*}{ Rhabdomyosarcoma $(n=82)$} & Total Positive & 0 & 4 & 1 \\
\hline & Total Cases & 27 & 26 & 36 \\
\hline & $\%$ Positive & 0.0 & 15.4 & 2.8 \\
\hline \multirow[t]{3}{*}{ Alveolar $(n=18)$} & Total Positive & 0 & 1 & 0 \\
\hline & Total Cases & 1 & 4 & 3 \\
\hline & $\%$ Positive & 0.0 & 25.0 & 0.0 \\
\hline \multirow[t]{3}{*}{ Embryonal $(n=19)$} & Total Positive & 0 & 0 & 0 \\
\hline & Total Cases & 7 & 8 & 6 \\
\hline & $\%$ Positive & 0.0 & 0.0 & 0.0 \\
\hline \multirow[t]{3}{*}{ Pleomorphic $(n=9)$} & Total Positive & 0 & 0 & 0 \\
\hline & Total Cases & 5 & 1 & 7 \\
\hline & $\%$ Positive & 0.0 & 0.0 & 0.0 \\
\hline \multirow[t]{3}{*}{ Other/Unknown $(n=36)$} & Total Positive & 0 & 3 & 1 \\
\hline & Total Cases & 14 & 12 & 20 \\
\hline & $\%$ Positive & 0.0 & 25.0 & 5.0 \\
\hline
\end{tabular}

(Continued) 


\begin{tabular}{|c|c|c|c|c|}
\hline \multirow{2}{*}{ Histology" } & & \multicolumn{3}{|c|}{ Gene $^{* *}$} \\
\hline & & \multirow{2}{*}{$\begin{array}{l}\text { ISH cMET } \\
0\end{array}$} & \multirow{2}{*}{$\begin{array}{l}\text { ISH EGFR } \\
1\end{array}$} & \multirow{2}{*}{$\begin{array}{l}\text { ISH HER2 } \\
0\end{array}$} \\
\hline $\operatorname{SFT}(n=56)$ & Total Positive & & & \\
\hline & Total Cases & 11 & 28 & 18 \\
\hline & $\%$ Positive & 0.0 & 3.6 & 0.0 \\
\hline \multirow[t]{3}{*}{ Synovial sarcoma $(n=70)$} & Total Positive & 1 & 0 & 0 \\
\hline & Total Cases & 19 & 31 & 21 \\
\hline & $\%$ Positive & 5.3 & 0.0 & 0.0 \\
\hline \multirow[t]{3}{*}{ UPS $(n=166)$} & Total Positive & 2 & 30 & 0 \\
\hline & Total Cases & 54 & 76 & 66 \\
\hline & $\%$ Positive & 3.7 & 39.5 & 0.0 \\
\hline \multirow[t]{3}{*}{ Other $(n=454)$} & Total Positive & 4 & 28 & 1 \\
\hline & Total Cases & 156 & 182 & 177 \\
\hline & $\%$ Positive & 2.6 & 15.4 & 0.6 \\
\hline
\end{tabular}

DSRCT $=$ desmoplastic small round cell tumor, $\mathrm{ESS}=$ endometrial stromal sarcoma, LMS = leiomyosarcoma, UPS/MFH = undifferentiated pleomorphic sarcoma/malignant fibrous histiocytoma, MPNST = malignant peripheral nerve sheath tumor, PEComa = perivascular epithelioid cell tumor, SFT = solitary fibrous tumor,

*Histologies with no amplification of genes tested: alveolar soft part sarcoma, chordoma, desmoid, epithelioid hemangioendothelioma, epithelioid sarcoma.

${ }^{* *}$ PIK3CA - 3/9 cases amplified( LMS, osteosarcoma, other); TOPO2A - 2/118 cases amplified(LMS, other); cMYC - 1/18 cases amplified(osteosarcoma); ALK - 1/65 cases amplified (LMS).

Anthracylines have served as a cornerstone for the treatment of sarcomas for decades. Response rates to single agent doxorubicin range from $9 \%$ to $27 \%$ [31, 32], with clinical benefit in approximately $60 \%$ of patients [33]. The mechanisms related to the lack of benefit seen in some patients are not entirely clear. TOPO2A is an important target for anthracyclines and its inhibition leads to double-strand DNA breaks and cell death. In breast cancer, TOPO2A protein expression by IHC has been correlated with response to anthracyclines [34], however this has been confounded by the co-expression of HER2. In sarcoma, a retrospective study of TOPO2A expression in 78 patients who underwent neoadjuvant therapy with an anthracycline, found that histologic response was positively correlated with high expression of TOPO2A [10]. This finding requires prospective validation. Although previous work has suggested overexpression of HER2 in approximately $50 \%$ of synovial sarcomas [26], none of the sarcoma specimens in this series co-expressed HER2. These findings also suggest that TOPO2A protein expression is not controlled by gene copy number, but through RNA or post-translational mechanisms. Our analysis also found an association between TOPO2A overexpression by IHC and TP53 mutation status. Indeed, in some studies, breast tumors containing TP53 mutations are exquisitely sensitive to anthracycline based therapy
[35]. It has been postulated and shown in TP53 wild type xenograft models that upon treatment with doxorubicin, there is induction of a senescent phenotype leading to cell cycle arrest and subsequent resistance to treatment $[36,37]$. This mutational status could therefore serve as a biomarker for sensitivity to anthracyclines in sarcoma and requires further study.

SPARC is a serum albumin-binding glycoprotein secreted by endothelial cells. It is hypothesized that tumoral SPARC could serve as a biomarker for sensitivity to the albumin-bound nanopartical of paclitaxel NABpaclitaxel. In pancreatic cancer, SPARC overexpression was correlated with response rate to nab-paclitaxel and prolonged progression free survival [38]. Clinically, angiosarcomas and EHE are essentially the only sarcoma subtypes where single agent taxanes are of benefit [39]. Though, SPARC expression has been assessed in sarcoma specimens, only 1 case of angiosarcoma has previously been evaluated and was found to have high expression of SPARC [12]. In our study, interestingly, SPARC was overexpressed in angiosarcoma, chondrosarcoma and EHE. A clinical trial of nab-paclitaxel in a wide variety of bone and soft tissue sarcomas did not meet its primary endpoint. However, this study did not assess for SPARC status or include patients with angiosarcoma or EHE [40]. MGMT gene silencing through promotor 


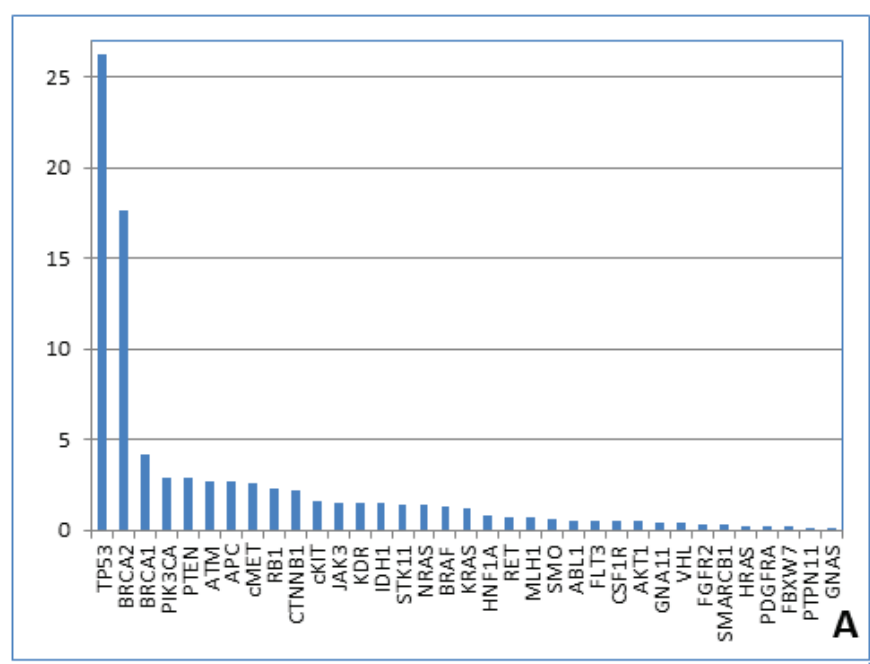

\begin{tabular}{|c|c|c|c|c|}
\hline Gene & Alteration & Exon & Frequency & Histology (N) \\
\hline BRAF & V6O0E & 15 & 6 & $\begin{array}{l}\text { Liposarcoma(unknown/other [1]), } \\
\text { angiosarcoma (1), MPNST (1), other (3) }\end{array}$ \\
\hline cKIT & T67S & 2 & 2 & UPS (1), synovial sarcoma (1) \\
\hline CMET & T1010I & 14 & 6 & $\begin{array}{l}\text { Angiosarcoma (1), LMS (1), myxoid } \\
\text { liposarcoma (1), pleomorphic liposarcoma } \\
\text { (1), ESS (1), epithelioid sarcoma (1) }\end{array}$ \\
\hline CMET & E1680 & 2 & 6 & $\begin{array}{l}\text { Mesenchymal chondrosarcoma (1), } \\
\text { comventional chondrosarcoma (1), LMS (2), } \\
\text { Other (2) }\end{array}$ \\
\hline CTNNB1 & S4SF, T41A & 3 & 13 & Desmoid (12), other (1) \\
\hline IDH1 & $\begin{array}{l}\text { R132C, R132S, } \\
\text { R132L }\end{array}$ & 4 & 6 & $\begin{array}{l}\text { Conventional chondrosarcoma (3), } \\
\text { chondrosarcoma (unknown/other [1]), UPS } \\
\text { (1), other (1) }\end{array}$ \\
\hline KRAS & $612 \mathrm{C}$ & 2 & 2 & Giant cell tumor (2) \\
\hline KRAS & $612 \mathrm{~V}$ & 2 & 5 & Fibrosarcoma (1), MPNST (1), other (3) \\
\hline NRAS & G12 & 2 & 5 & Angiosarcoma (4), ESS (1) \\
\hline NRAS & Q61 & 3 & 3 & Other (3) \\
\hline PIK3CA & H1047R & 20 & 5 & $\begin{array}{l}\text { Myxoid liposarcoma (3), fibrosarcoma (1), } \\
\text { other (1) }\end{array}$ \\
\hline PIK3CA & $\begin{array}{l}\text { ES42Q, ES45K, } \\
\text { OS46K }\end{array}$ & 9 & 12 & $\begin{array}{l}\text { LMS (1), pleomorphic liposarcoma (1), UPS } \\
\text { (2), rhabdomyosarcoma (unknown/other } \\
\text { [2]), other (3), chordoma (2), ESS (1) }\end{array}$ \\
\hline PIK3CA & R88Q & 1 & 1 & Other (1) \\
\hline PIK3CA & N345K & 4 & 1 & UPS (1) \\
\hline
\end{tabular}
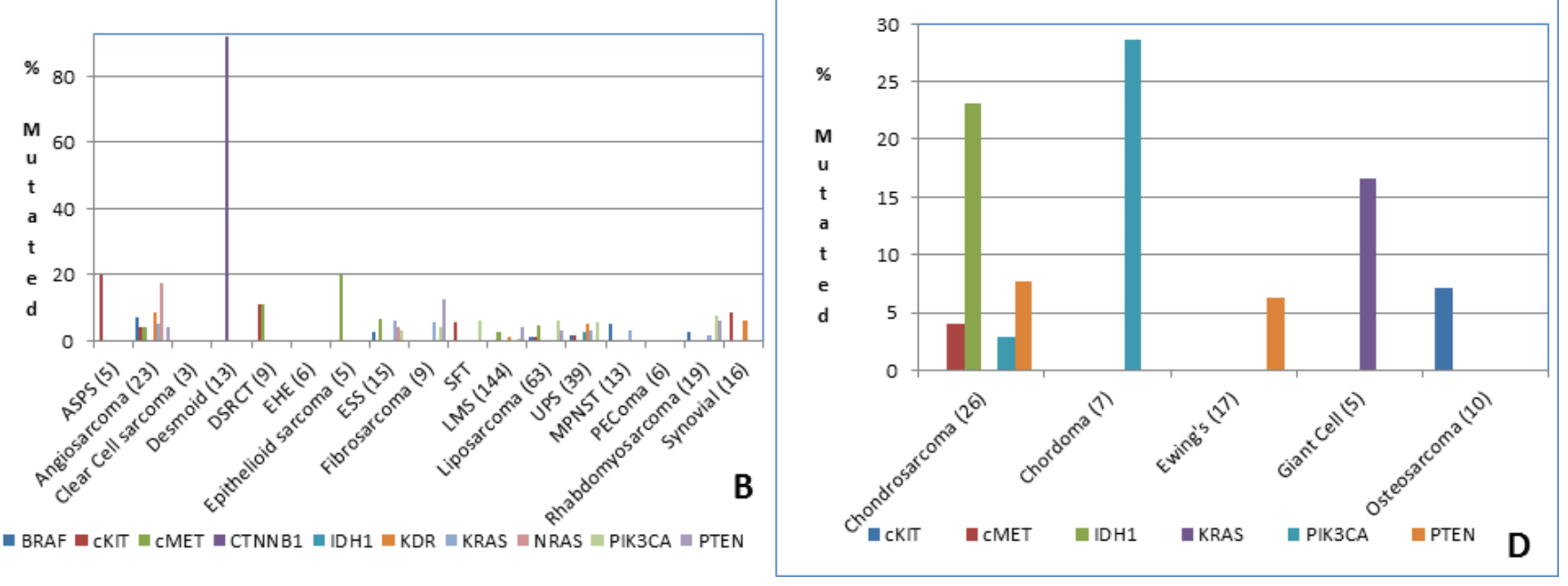

Figure 2: DNA sequencing in sarcoma. A. Most frequent mutation (\%) B. Mutation type C. Mutations in STS D. Mutations in bone sarcoma. ASPS = alveolar soft part sarcoma, DSRCT = desmoplastic small round cell tumor, ESS = endometrial stromal sarcoma, $\mathrm{SFT}=$ solitary fibrous tumor, $\mathrm{LMS}=$ leiomyosarcoma, UPS $=$ undifferentiated pleomorphic sarcoma, MPNST = malignant peripheral nerve sheath tumor, PEComa = perivascular epithelioid cell tumor.

methylation confers sensitivity to the alkylating agents in glioblastoma [41]. Previous work has suggested that $20 \%$ of STS have MGMT loss by IHC and $8 \%$ have both promoter methylation and negative IHC nuclear staining. Response to temozolomide has been noted in a patient with a resistant undifferentiated high grade sarcoma whose tumor demonstrated MGMT promoter methylation and protein loss by IHC [42].

Targeted therapy has improved outcomes of patients with solid tumors such as lung and kidney cancer. Pazopanib remains the only FDA approved targeted therapy in STS, as other trials of novel agents have largely been unsuccessful when tested in a broad- range of sarcomas. Biomarkers predicting response would therefore be of utility. The PI3-kinase pathway is of great interest in sarcoma and cancer in general. Previous studies have shown PTEN partial genomic loss and loss of protein expression in LMS. Gibault and colleagues showed that partial loss of PTEN gene by array-comparative genomic hybridization (a-CGH) occurs in 39\% of sarcomas with complex genomics and that this may be sufficient for a pathologic phenotype [14]. On the other hand, PTEN promoter methylation appears to be an uncommon event and may not play a major role in down-regulation of PTEN expression $[14,15]$. In our study PTEN loss was most notable in the chordomas, epithelioid sarcomas, alveolar rhabdomyosarcomas and osteosarcomas. Though loss was noted in the LMS cohort, this was only seen in about $35 \%$ of the cases. Disappointingly, targeting this pathway with mTOR inhibitors has been unsuccessful clinically [43], possibly owing to the fact that preselection of tumors with alterations in the PI3-kinase pathway was not required for trial enrollment. Overall, the rate of PIK3CA and PTEN mutations was low in our series. PTEN mutations were most commonly seen in chondrosarcomas (7.7\%), myxoid liposarcomas (16.7\%), 
Table 2: Concordance across platforms

\begin{tabular}{|c|c|c|c|c|}
\hline & $\begin{array}{l}\mathbf{N} \text { positive } \\
\text { concordance/ } \mathbf{N}\end{array}$ & $\mathrm{IHC}+(\%)$ & FISH amplified (\%) & Mutation (\%) \\
\hline $\mathrm{cKIT}$ & $0 / 23$ & $65 / 1441(4.5)$ & NA & 12/749 (1.6) All VUS* \\
\hline cMET & \begin{tabular}{|l}
$1 / 751(\mathrm{IHC} / \mathrm{ISH})$ \\
$0 / 442(\mathrm{ISH} / \mathrm{NGS})$ \\
$1 / 567(\mathrm{IHC} / \mathrm{NGS})$
\end{tabular} & $43 / 970(4.4)$ & 20/761 (2.6) & $\begin{array}{l}\text { 15/588 (2.6) } \\
\text { All VUS* }\end{array}$ \\
\hline EGFR & $\begin{array}{l}\text { 2/45 (IHC/ISH) } \\
0 / 16 \text { (ISH/NGS) } \\
0 / 11 \text { (IHC/NGS) }\end{array}$ & $80 / 217$ (36.9) & $181 / 1072(16.9)$ & $0 / 608(0)$ \\
\hline HER2 & $\begin{array}{l}\text { 0/910 (IHC/ISH) } \\
0 / 518 \text { (ISH/NGS) } \\
0 / 555 \text { (IHC/NGS }\end{array}$ & $\begin{array}{l}1 / 2409(0.04) \\
{ }^{*} \text { Case with HER2 } \\
\text { overexpression was } \\
\text { not tested on other } \\
\text { platforms }\end{array}$ & 9/925 (1.0) & $0 / 573(0)$ \\
\hline PDGFR & NA & $135 / 610(22.1)$ & NA & $1 / 581(0)$ \\
\hline PTEN & 8/539 (IHC/NGS) & Loss 910/2358 (38.6) & NA & $\begin{array}{l}\text { 16/557(15 cases) (3.2) } \\
\text { *8/15 cases with } \\
\text { PTEN mutation had } \\
\text { PTEN loss } \\
{ }^{*} 7 / 15 \text { cases with } \\
\text { mutation were VUS } \\
\text { without PTEN loss }\end{array}$ \\
\hline TOPO2A & 0/36(IHC/FISH) & $1117 / 2114(52.8)$ & $2 / 118(1.7)$ & NA \\
\hline
\end{tabular}

${ }^{*}$ VUS $=$ variant of unknown significance

Table 3: Biomarker associations

\begin{tabular}{|c|c|c|c|c|c|c|c|c|}
\hline & $\begin{array}{l}\text { PTEN } \\
\text { Loss IHC }\end{array}$ & $\begin{array}{l}\text { TOPO2 } \\
\text { IHC+ }\end{array}$ & $\begin{array}{l}\text { PTEN } \\
\text { MT }\end{array}$ & $\begin{array}{l}\text { cMET } \\
\text { MT }\end{array}$ & IDH MT & $\begin{array}{l}\text { CTNNB1 } \\
\text { MT }\end{array}$ & APC MT & $\begin{array}{l}\text { KRAS } \\
\text { MT }\end{array}$ \\
\hline TP53wt & \begin{tabular}{|l}
$56 / 414$ \\
$(13.5 \%)$
\end{tabular} & \begin{tabular}{|l|}
$226 / 396$ \\
$(57.1 \%)$
\end{tabular} & $\begin{array}{l}9 / 407 \\
(2.2 \%)\end{array}$ & \begin{tabular}{|l|}
$8 / 427$ \\
$(1.9 \%)$
\end{tabular} & $\begin{array}{l}5 / 430 \\
(1.2 \%)\end{array}$ & $\begin{array}{l}13 / 430 \\
(3.0 \%)\end{array}$ & $\begin{array}{l}11 / 429 \\
(2.6 \%)\end{array}$ & $\begin{array}{l}7 / 429 \\
(1.6 \%)\end{array}$ \\
\hline TP53 mutated & $\begin{array}{l}22 / 135 \\
(16.3 \%)\end{array}$ & $\begin{array}{l}115 / 134 \\
(85.8 \%)\end{array}$ & $\begin{array}{l}5 / 127 \\
(3.9 \%)\end{array}$ & \begin{tabular}{|l}
$6 / 136$ \\
$(4.4 \%)$
\end{tabular} & $\begin{array}{l}4 / 136 \\
(2.9 \%)\end{array}$ & $0 / 136(0)$ & $\begin{array}{l}5 / 135 \\
(3.7 \%)\end{array}$ & $\begin{array}{l}1 / 135 \\
(0.7 \%)\end{array}$ \\
\hline$P$ value & 0.48 & 0.0001 & 0.34 & 0.11 & 0.23 & 0.045 & 0.55 & 0.69 \\
\hline
\end{tabular}

Table 4: Biomarker associations

\begin{tabular}{|l|l|l|ll|}
\hline \multicolumn{1}{l}{ TP53 MT (\%) } & \multicolumn{1}{l}{ PTEN Loss IHC (\%) } & TOPO2 IHC+ (\%) & PTEN MT (\%) \\
\hline PIK3CA mutated & $5 / 20(25.0 \%)$ & $3 / 22(13.6 \%)$ & $14 / 20(70.0 \%)$ & $2 / 18(11.1 \%)$ \\
\hline PIK3CA WT & $140 / 533(26.3 \%)$ & $143 / 781(18.3 \%)$ & $367 / 567(64.7 \%)$ & $13 / 529(2.5 \%)$ \\
\hline$P$ value & 1.0 & 0.78 & 0.81 & 0.08 \\
\hline
\end{tabular}

Ewing's sarcoma and rhabdomyosarcoma (6.3\%). Previous work has demonstrated PIK3CA mutations in $14-18 \%$ of myxoid and round cell liposarcoma $[3,5]$. In our series, PIK3CA mutations were most commonly seen in myxoid liposarcoma $(23.5 \%)$, as well as in rhabdomyosarcoma
(7.4\%), solitary fibrous tumor (6.3\%) and UPS (5.5\%). We had 1 case of myxoid liposarcoma with both PTEN loss by IHC and PIK3CA mutation, not previously described in the literature [3]. We also detected pathogenic PTEN mutations in angiosarcoma specimens, which have not 
previously been described in the literature [4]. Finally, our data suggests the co-existence of PTEN and TP53 mutations, as well as TP53 mutations and PTEN loss in sarcoma specimens. Indeed, it is known that there is considerable crosstalk between these two tumor suppressor genes $[44,45]$. The prognostic effect of this phenomenon is not known in sarcoma; therefore validation against phase III clinical data is needed. Our data also confirmed the rate of ER positivity in LMS. A phase II study of the aromatase inhibitor letrozole in these patients has demonstrated clinical benefit [46]. On the other hand, though PDGFRA was overexpressed in many tumors consistent with previous work [16-18], phase II studies of imatinib in PDGFR- positive sarcomas failed to show benefit $[18,47]$.

Newer immunotherapies have demonstrated success in melanoma, kidney cancers and lung cancers, but have not yet been evaluated in sarcoma [48]. Currently of most interest are the PD-1 and PD-L1 inhibitors. Previous work has shown that both PD-1 and PD-L1 positivity were independent prognostic indicators for OS and EFS in sarcoma [22]. PD-1 positive tumor-infiltrating lymphocytes and PD-L1 expression in tumor cells were seen in $65 \%$ and $58 \%$ of STS cases respectively. Uncertainty remains as to the effectiveness of PD-L1 expression in tumor as a biomarker for sensitivity to the checkpoint inhibitors as patients with low or no expression of PD-L1 may still have a response and/or survival benefit [49]. PD-L1 expression was noted in the majority of chondrosarcomas, LMS, UPS and liposarcoma in our series.

Protein expression is a semi-quantitative, inclusive end-point. Changes in protein level may be a result of mutations, increased gene copy number, changes in gene expression, or biochemical responses within the cell to environmental factors. In other solid tumors, there is concordance between protein expression by IHC and mutation or gene amplification as in the cases of cKIT and HER2 respectively [50, 51]. However, discordance has been documented across solid tumors for cMET, TOP2A, and PTEN [30], proposed to be due to downstream modulation of protein expression levels. There was relatively low concordance across platforms in our series, suggesting that protein levels of cKIT, cMET and TOPO2A may be regulated at the RNA or post translational level. On the other hand, IHC for certain proteins such as EGFR, is known to be unreliable, owing the antibodies and scoring system used [23]. In fact, a prior clinical trial of gefitinib in synovial sarcomas with enrollment limited to patients overexpressing EGFR by IHC was without responses to this agent. Twentyone percent of patients did have stable disease [52]. In addition, no EGFR mutations were detected in our sarcomas, consistent with other series [24]. Our data set suggests that EGFR gene amplification could be used instead of IHC as a biomarker for sensitivity to the EGFR inhibitors. This gene amplification was most notable in DSRCT, LMS, MPNST, osteosarcoma and UPS.
There are several limitations to this analysis. Though all specimens underwent pathology review to confirm a diagnosis of sarcoma, the specific subtype was determined by the submitting institution's pathologist. This is problematic for non-specific diagnoses such as fibrosarcoma, which may in fact represent a heterogenous group of tumors. The lack of concordance across platforms observed in this series is of interest. This may be attributed, as in the case of EGFR, to the unreliability of IHC. In this case, it seems that copy number analysis may be more useful. On the other hand, the lack of concordance between $c K I T$ activating mutations and increased protein expression suggests an inadequacy in the methodology used to detect these genetic alterations. In fact, there was a low frequency of actionable mutations detected in this series overall, suggesting that Hotspot analysis has limited scope in sarcoma and techniques such as whole exome sequencing should be explored. Despite these limitations in technique, there were a few noteworthy observations, including alteration of the RAF/RAS/MEK/ERK pathway in angiosarcoma. Finally, this analysis does not include details on treatment history and/or patient outcomes which would be useful as a first step in validation of these potential biomarkers.

These data represent a large analysis of molecular alterations by sarcoma subtype. Although there are limitations of this analysis, this data catalogue provides the groundwork for future clinical trials in sarcoma and highlights the need for the sarcoma community to prospectively validate tumor biomarkers in our clinical trials.

\section{MATERIALS AND METHODS}

Data from sarcoma specimens profiled on at least one platform by Caris Life Sciences from January 11, 2006 through July 31, 2014 were included. Formalin fixed paraffin-embedded (FFPE) sarcoma samples were sent for analysis from treating physicians around the world (59 countries). The specific sarcoma histology was extracted from paperwork submitted by the treating physician. GIST was excluded, to limit the analysis to sarcomas without known therapeutic targets. Tumors were initially verified by a board certified pathologist for sufficient tumor presence and to confirm the diagnosis of sarcoma. Any sample reviewed and determined not to be a sarcoma was excluded from this cohort. Samples were subsequently analyzed using one or more of the profiling platforms as described below. Biomarkers for analysis were selected based on their potential to be targeted therapeutically and/or based on clinical evidence of a utility in other solid tumors.

\section{Immunohistochemistry (IHC)}

Protein expression was determined by IHC analysis, using commercially available detection kits and automated staining techniques (Benchmark XT, Ventana, and 
AutostainerLink 48, Dako) [53]. Antibodies used included: androgen receptor (AR), topoisomerases 1 and 2 (TOPO1, TOPO2A) (Leica Biosystems); ER, PR, cMET, HER2 (Ventana); cKIT, epidermal growth factor receptor (EGFR), phosophatase and tensin homolog (PTEN) (Dako), O(6)methylguanine-methyltransferase (MGMT), P-glycoprotein (PGP) (Invitrogen); transducin-like enhancer of split 3 (TLE3, Santa Cruz); ribonucleotide reductase M1 (RRM1, Protein Tech); SPARC (monoclonal, R\&D Systems; polyclonal, Exalpha), tubulin beta-3 chain (TUBB3) (Covance), Excision Repair Cross-Complementation Group 1 (ERCC1, (Abcam), platelet derived growth factor receptor alpha (PDGFRA, Thermo), Programmed cell death protein 1 (PD-1) and Programmed death-ligand 1 (PD-L1) (BD Pharmingen and R\&D Systems). IHC thresholds previously validated in other cancers were used, as cutoffs are not established in sarcoma (Appendix 1).

\section{In situ hybridization}

Fluorescent in-situ hybridization (FISH) was used for evaluation of the HER-2/neu [HER-2/CEP17 probe; HER2/CEP17 ratio $>2.2$ was considered amplified], EGFR
[EGFR/CEP7 probe EGFR/CEP7 ratio $\geq 2$, or $\geq 15$ EGFR copies per cell in $\geq 10 \%$ of analyzed cells was considered amplified], TOP $2 A$ [TOP2/CEP17 probe; TOP2A/CEP17 ratio $\geq 2.0$ was considered amplified], $c M E T$ [cMET/CEP7 probe; cMET/CEP7 ratio $\geq 5$ was considered amplified], $A L K$ [Break Apart Probe; ALK rearrangements, either inversion or translocation of the ALK gene at $2 \mathrm{p} 23$, were identified by separation of the fusion signals into one red and one green signal], $c M Y C$ [cMYC/CEP8 probe; $\mathrm{cMYC/}$ CEP8 ratio $\geq 2$ was considered amplified], and $P I K 3 C A$ [PIK3CA/CEP3 probe; PIK3CA/CEP3 $\geq 3$ was considered amplified] (Abbott Molecular/Vysis). HER-2/neu and $c M E T$ status were more recently evaluated by chromogenic in-situ hybridization (INFORM HER-2 Dual ISH DNA Probe Cocktail; commercially available cMET and chromosome 7 DIG probe; Ventana), and used the same scoring system as for FISH.

\section{Mutational analysis}

\section{Next-Generation Sequencing (NGS)}

Direct sequence analysis was performed on genomic DNA isolated from FFPE tumor samples using the Illumina

\section{Appendix 1: IHC Thresholds}

\begin{tabular}{|c|c|}
\hline Protein & Threshold for Overexpression (Intensity and \%expression in tumor) \\
\hline AR & $\geq 1+$ and $\geq 10 \%$ \\
\hline $\mathrm{cKIT}$ & $\geq 2+$ and $\geq 30 \%$ \\
\hline cMET & $\geq 2+$ and $\geq 50 \%$ \\
\hline EGFR & $2+$ and $=10 \%$ or $1+$ and $<10 \%$; current $=$ H-score,$<200$ or $\geq 200$ \\
\hline ER & $\geq 1+$ and $\geq 10 \%$ \\
\hline PR & $\geq 1+$ and $\geq 10 \%$ \\
\hline HER2 & $\geq 3+$ and $>10 \%$ \\
\hline PD-1 & $\geq 1+($ TIL Count/High power field with $40 \mathrm{x}$ objective $)$ \\
\hline PD-L1 & $\geq 2+$ and $\geq 5 \%$ \\
\hline PDGFRA & $\geq 2+$ and $30 \%$ \\
\hline PGP & $\geq 1+$ and $\geq 10 \%$ \\
\hline SPARC & $\geq 2+$ and $\geq 30 \%$ \\
\hline TLE3 & $\geq 2+$ and $\geq 30 \%$ \\
\hline TOPO1 & $\geq 2+$ and $\geq 30 \%$ \\
\hline TOP2A & $\geq 1+$ and $\geq 10 \%$ \\
\hline TUBB3 & $\geq 2+$ and $\geq 30 \%$ \\
\hline Protein & Threshold for Loss (Intensity and \%expression in tumor) \\
\hline ERCC1 & $<2+$ or $\leq 3+$ and $<10 \%$ or $=2+$ and $<50 \%$ \\
\hline MGMT & $=0+$ or $\leq 35 \%$ \\
\hline PTEN & $=0+$ or $\leq 50 \%$ \\
\hline RRM1 & $<2+$ or $<50 \%$ \\
\hline
\end{tabular}


MiSeq platform. Average sequencing depth was $>1000 \mathrm{X}$. Specific regions of 47 genes were amplified using the Illumina TruSeq Amplicon Cancer Hotspot panel.

\section{Sanger sequencing}

Prior to the availability of CLIA certified NGS, mutation analysis by Sanger sequencing included selected regions of BRAF, KRAS, cKIT, EGFR, and PIK3CA genes and was performed by using M13-linked PCR primers designed to amplify targeted sequences. PCR products were bi-directionally sequenced using the BigDye Terminator v1.1 chemistry, analyzed using the 3730 DNA Analyzer (Applied Biosystems). Sequence traces were analyzed using Mutation Surveyor software v3.25 (Soft Genetics).

\section{Statistical analysis}

The patient population and profiling data were characterized using standard descriptive statistics. When comparing data across the subtypes, groups with less than 10 cases were not considered. For chemotherapy protein biomarkers, overexpression or loss in at least $60 \%$ of samples in a particular subtype were considered clinically significant (mean selected as cutoff). For targeted therapy protein biomarkers, overexpression in at least $17.5 \%$ of cases was considered clinically relevant (mean selected as cutoff). Concordance across platforms was determined using Cohen's Kappa.

\section{CONFLICTS OF INTEREST}

W. Wen, W. Chen, S.Z. Millis, Z. Gatalica and S. Reddy are employees of Caris Life Sciences. B.A. Van Tine is a consultant/advisory board member for Caris Life Sciences. There are no potential conflicts of interest for the other authors.

\section{Editorial note}

This paper has been accepted based in part on peer-review conducted by another journal and the authors' response and revisions as well as expedited peer-review in Oncotarget.

\section{REFERENCES}

1. Italiano A, Mathoulin-Pelissier S, Cesne AL, Terrier P, Bonvalot S, Collin F, Michels JJ, Blay JY, Coindre JM, Bui B. Trends in survival for patients with metastatic soft-tissue sarcoma. Cancer. 2011; 117:1049-1054.

2. Taylor BS, Barretina J, Maki RG, Antonescu CR, Singer S, Ladanyi M. Advances in sarcoma genomics and new therapeutic targets. Nat Rev Cancer. 2011; 11:541-557.
3. Demicco EG, Torres KE, Ghadimi MP, Colombo C, Bolshakov S, Hoffman A, Peng T, Bovee JV, Wang WL, Lev D, Lazar AJ. Involvement of the PI3K/Akt pathway in myxoid/round cell liposarcoma. Mod Pathol. 2012; 25:212-221.

4. Italiano A, Chen CL, Thomas R, Breen M, Bonnet F, Sevenet N, Longy M, Maki RG, Coindre JM, Antonescu CR. Alterations of the p53 and PIK3CA/AKT/ mTOR pathways in angiosarcomas: a pattern distinct from other sarcomas with complex genomics. Cancer. 2012; 118:5878-5887.

5. Barretina J, Taylor BS, Banerji S, et al. Subtype-specific genomic alterations define new targets for soft-tissue sarcoma therapy. Nat Genet. 2010; 42:715-721.

6. Cote G, Butrynski J, Shen J, et al. Genomic analysis of over 400 sarcomas. Connective Tissue Oncology Society. 2013.

7. Davies C, Godwin J, Gray R, Clarke M, Cutter D, Darby S, McGale P, Pan H.C, Taylor C, Wang Y.C, Dowsett M, Ingle $\mathrm{J}$, Peto R. Relevance of breast cancer hormone receptors and other factors to the efficacy of adjuvant tamoxifen: patient-level meta-analysis of randomised trials. Lancet. 2011; 378:771-784.

8. Slamon D, Eiermann W, Robert N, et al. Adjuvant trastuzumab in HER2-positive breast cancer. N Engl J Med. 2011; 365:1273-1283.

9. Schenone AD, Van Tine BA. Prospective Biomarkers for Chemotherapy Selection in Sarcoma. ESUN. 2014; 11:4.

10. Rodrigo RS, Nathalie A, Elodie T, et al. Topoisomerase II-alpha protein expression and histological response following doxorubicin-based induction chemotherapy predict survival of locally advanced soft tissues sarcomas. Eur J Cancer. 2011; 47:1319-1327.

11. Brase JC, Schmidt M, Fischbach T, Sultmann H, Bojar H, Koelbl H, Hellwig B, Rahnenfuhrer J, Hengstler JG, Gehrmann MC. ERBB2 and TOP2A in breast cancer: a comprehensive analysis of gene amplification, RNA levels, and protein expression and their influence on prognosis and prediction. Clin Cancer Res. 2010; 16:2391-2401.

12. Morgan SS, Nagle RB, Cranmer LD. Serum protein acidic and rich in cysteine (SPARC) as a prognostic marker in soft tissue sarcomas. Clin Sarcoma Res. 2014; 4:2.

13. Jakob J, Hille M, Sauer C, Strobel P, Wenz F, Hohenberger P. O6-methylguanine-DNA methyltransferase (MGMT) promoter methylation is a rare event in soft tissue sarcoma. Radiat Oncol. 2012; 7:180.

14. Gibault L, Ferreira C, Perot G, Audebourg A, Chibon F, Bonnin S, Lagarde P, Vacher-Lavenu MC, Terrier P, Coindre JM, Aurias A. From PTEN loss of expression to RICTOR role in smooth muscle differentiation: complex involvement of the mTOR pathway in leiomyosarcomas and pleomorphic sarcomas. Mod Pathol. 25:197-211. 
15. Kawaguchi K, Oda Y, Saito T, Takahira T, Yamamoto H, Tamiya S, Iwamoto Y, Tsuneyoshi M. Genetic and epigenetic alterations of the PTEN gene in soft tissue sarcomas. Hum Pathol. 2005; 36:357-363.

16. Hoffman A, Ghadimi MP, Demicco EG, et al. Localized and metastatic myxoid/round cell liposarcoma: clinical and molecular observations. Cancer. 2013; 119:1868-1877.

17. Lusby K, Savannah KB, Demicco EG, et al. Uterine leiomyosarcoma management, outcome, and associated molecular biomarkers: a single institution's experience. Ann Surg Oncol. 2013; 20:2364-2372.

18. Grignani G, Palmerini E, Stacchiotti S, Boglione A, Ferraresi V, Frustaci S, Comandone A, Casali PG, Ferrari S, Aglietta M. A phase 2 trial of imatinib mesylate in patients with recurrent nonresectable chondrosarcomas expressing platelet-derived growth factor receptor-alpha or -beta: An Italian Sarcoma Group study. Cancer. 2011; 117:826-831.

19. Leitao MM, Jr., Hensley ML, Barakat RR, Aghajanian C, Gardner GJ, Jewell EL, O'Cearbhaill R, Soslow RA. Immunohistochemical expression of estrogen and progesterone receptors and outcomes in patients with newly diagnosed uterine leiomyosarcoma. Gynecol Oncol. 2012; 124:558-562.

20. Bodner K, Bodner-Adler B, Kimberger O, Czerwenka K, Leodolter S, Mayerhofer K. Estrogen and progesterone receptor expression in patients with uterine leiomyosarcoma and correlation with different clinicopathological parameters. Anticancer Research. 2003; 23:729-732.

21. Kelley TW, Borden EC, Goldblum JR. Estrogen and progesterone receptor expression in uterine and extrauterine leiomyosarcomas: an immunohistochemical study. Appl Immunohistochem Mol Morphol. 2004; $12: 338-341$.

22. Ryul Kim J, Jae Moon Y, Sang Kwon K, Sang Bae J, Wagle S, Min Kim K, Sung Park H, Lee H, Sung Moon W, Ja Chung M, Jae Kang M, Yun Jang K. Tumor Infiltrating PD1-Positive Lymphocytes and the Expression of PD-L1 Predict Poor Prognosis of Soft Tissue Sarcomas. PLoS One. 2013; 8:e82870.

23. Kersting C, Packeisen J, Leidinger B, Brandt B, von Wasielewski $\mathrm{R}$, Winkelmann $\mathrm{W}$, van Diest PJ, Gosheger G, Buerger H. Pitfalls in immunohistochemical assessment of EGFR expression in soft tissue sarcomas. J Clin Pathol. 2006; 59:585-590.

24. Holtkamp N, Malzer E, Zietsch J, Okuducu AF, Mucha J, Mawrin C, Mautner VF, Schildhaus HU, von Deimling A. EGFR and erbB2 in malignant peripheral nerve sheath tumors and implications for targeted therapy. Neuro Oncol. 2008; 10:946-957.

25. Du X, Yang J, Ylipaa A, Zhu Z. Genomic amplification and high expression of EGFR are key targetable oncogenic events in malignant peripheral nerve sheath tumor. J Hematol Oncol. 2013; 6:93.
26. Thomas DG, Giordano TJ, Sanders D, Biermann S, Sondak VK, Trent JC, Yu D, Pollock RE, Baker L. Expression of receptor tyrosine kinases epidermal growth factor receptor and HER-2/neu in synovial sarcoma. Cancer. 2005; 103:830-838.

27. Hirota S, Isozaki K, Moriyama $\mathrm{Y}$, et al. Gain-of-function mutations of c-kit in human gastrointestinal stromal tumors. Science. 1998; 279:577-580.

28. Bouchalova K, Trojanec R, Kolar Z, Cwiertka K, Cernakova I, Mihal V, Hajduch M. Analysis of ERBB2 and TOP2A gene status using fluorescence in situ hybridization versus immunohistochemistry in localized breast cancer. Neoplasma. 2006; 53:393-401.

29. Mueller RE, Parkes RK, Andrulis I, O’Malley FP. Amplification of the TOP2A gene does not predict high levels of topoisomerase II alpha protein in human breast tumor samples. Genes Chromosomes Cancer. 2004; 39:288-297.

30. O'Malley FP, Chia S, Tu D, Shepherd LE, Levine MN, Huntsman D, Bramwell VH, Andrulis IL, Pritchard KI. Topoisomerase II alpha protein and responsiveness of breast cancer to adjuvant chemotherapy with CEF compared to CMF in the NCIC CTG randomized MA.5 adjuvant trial. Breast Cancer Res Treat. 2011; 128:401-409.

31. Schoenfeld DA, Rosenbaum C, Horton J, Wolter JM, Falkson G, DeConti RC. A comparison of adriamycin versus vincristine and adriamycin, and cyclophosphamide versus vincristine, actinomycin-D, and cyclophosphamide for advanced sarcoma. Cancer. 1982; 50:2757-2762.

32. Judson I, Radford JA, Harris M, et al. Randomised phase II trial of pegylated liposomal doxorubicin (DOXIL/ CAELYX) versus doxorubicin in the treatment of advanced or metastatic soft tissue sarcoma: a study by the EORTC Soft Tissue and Bone Sarcoma Group. Eur J Cancer. 2001; 37:870-877.

33. Judson I, Verweij J, Gelderblom H, et al. Doxorubicin alone versus intensified doxorubicin plus ifosfamide for first-line treatment of advanced or metastatic soft-tissue sarcoma: a randomised controlled phase 3 trial. Lancet Oncology. 2014; 15:415-423.

34. Mukherjee A, Shehata M, Moseley P, Rakha E, Ellis I, Chan S. Topo2alpha protein expression predicts response to anthracycline combination neo-adjuvant chemotherapy in locally advanced primary breast cancer. Br J Cancer. 2010; 103:1794-1800.

35. Bertheau P, Turpin E, Rickman DS, et al. Exquisite sensitivity of TP53 mutant and basal breast cancers to a dose-dense epirubicin-cyclophosphamide regimen. PLoS Med. 2007; 4:e90.

36. Varna M, Lehmann-Che J, Turpin E, et al. p53 dependent cell-cycle arrest triggered by chemotherapy in xenografted breast tumors. Int J Cancer. 2009; 124:991-997. 
37. Jackson JG, Pant V, Li Q, Chang LL, Quintas-Cardama A, Garza D, Tavana O, Yang P, Manshouri T, Li Y, El-Naggar AK, Lozano G. p53-mediated senescence impairs the apoptotic response to chemotherapy and clinical outcome in breast cancer. Cancer Cell. 2012; 21:793-806.

38. Von Hoff D, Ramanathan R, Borad M, Laheru D, Smith L, Wood L, Korn R, Desai N, Iglesias J, Hidalgo M. SPARC correlation with response to gemcitabine $(G)$ plus nab-paclitaxel (nab-P) in patients with advanced metastatic pancreatic cancer: A phase I/II study. J Clin Oncol. 2009; 27:15s.

39. Penel N, Bui BN, Bay JO, et al. Phase II trial of weekly paclitaxel for unresectable angiosarcoma: the ANGIOTAX Study. J Clin Oncol. 2008; 26:5269-5274.

40. Butrynski J, Nadadur R, Jahan T, Chua V, Bautista Lazaro C, Chawla S. A Phase II study of nanoparticle albumin-bound (NAB) paclitaxel in the treatment of patients with unresectable or metastatic sarcoma. Cancer Sci. 2014; 105:812-7.

41. Hegi ME, Diserens AC, Gorlia T, et al. MGMT gene silencing and benefit from temozolomide in glioblastoma. N Engl J Med. 2005; 352:997-1003.

42. Tanaka H, Sasayama T, Nishihara M, Arai A, Kawamura A, Kanomata N, Itoh T, Kohmura E. Brain metastasis of undifferentiated sarcoma and response to temozolomide treatment. Case report. Neurol Med Chir (Tokyo). 2010; 50:689-693.

43. Okuno S, Bailey H, Mahoney MR, Adkins D, Maples W, Fitch T, Ettinger D, Erlichman C, Sarkaria JN. A phase 2 study of temsirolimus (CCI-779) in patients with soft tissue sarcomas: a study of the Mayo phase 2 consortium (P2C). Cancer. 2011; 117:3468-3475.

44. Said R, Ye Y, Hong D.S, Janku F, Fu S, Naing A, Wheler J.J, Kurzrock R, Thomas C, Palmer G.A, Hess K R, Aldape K, Tsimberidou A.M. Characteristics and survival of patients with advanced cancer and p53 mutations. Oncotarget. 2014; 5:3871-3879.

45. Freeman DJ, Li AG, Wei G, Li HH, Kertesz N, Lesche R, Whale AD, Martinez-Diaz H, Rozengurt N, Cardiff RD, Liu X, Wu H. PTEN tumor suppressor regulates p53 protein levels and activity through phosphatase-dependent and -independent mechanisms. Cancer Cell. 2003; 3:117-130.
46. George S, Feng Y, Manola J, Nucci M.R, Butrynski J.E, Morgan J.A, Ramaiya N, Quek R, Penson R.T, Wagner A.J, Harmon D, Demetri G.D, Krasner C. Phase 2 trial of aromatase inhibition with letrozole in patients with uterine leiomyosarcomas expressing estrogen and/or progesterone receptors. Cancer. 2014; 120:738-743.

47. Sugiura H, Fujiwara Y, Ando M, Kawai A, Ogose A, Ozaki T, Yokoyama R, Hiruma T, Ishii T, Morioka H, Mugishima H. Multicenter phase II trial assessing effectiveness of imatinib mesylate on relapsed or refractory KITpositive or PDGFR-positive sarcoma. J Orthop Sci. 2010; 15:654-660.

48. McDermott DF, Atkins MB. PD-1 as a potential target in cancer therapy. Cancer Med. 2013; 2:662-673.

49. Kefford R, Ribas A, Hamid O, et al. Clinical efficacy and correlation with tumor PD-L1 expression in patients (pts) with melanoma (MEL) treated with the anti-PD-1 monoclonal antibody MK-3475. J Clin Oncol. $2014 ; 32: 5$ s.

50. Rubin BP, Singer S, Tsao C, Duensing A, Lux M.L, Ruiz R, Hibbard M.K, Chen C J, Xiao S, Tuveson D.A. Demetri G.D, Fletcher C.D, Fletcher J.A. KIT activation is a ubiquitous feature of gastrointestinal stromal tumors. Cancer Res. 2001; 61:8118-8121.

51. Wolff AC, Hammond ME, Hicks DG, et al. Recommendations for human epidermal growth factor receptor 2 testing in breast cancer: American Society of Clinical Oncology/College of American Pathologists clinical practice guideline update. J Clin Oncol. 2013; 31:3997-4013.

52. Blay J, Le Cesne A, Whelan J, Van Oosterom A, Ray-Coquard I, Judson L, Hogendorn P, Marreaud S, Hermans C, Van Glabbeke M. Gefitinib in second line treatment of metastatic or locally advanced synovial sarcoma expressing HER1: A phase II trial of EORTC Soft Tissue and Bone Sarcoma Group. J Clin Oncol. 2006; 24:524s-524s.

53. Millis SZ, Bryant D, Basu G, Bender R, Vranic S, Gatalica Z, Vogelzang NJ. Molecular profiling of infiltrating urothelial carcinoma of bladder and nonbladder origin. Clin Genitourin Cancer. 2015; 13:e37-49. 